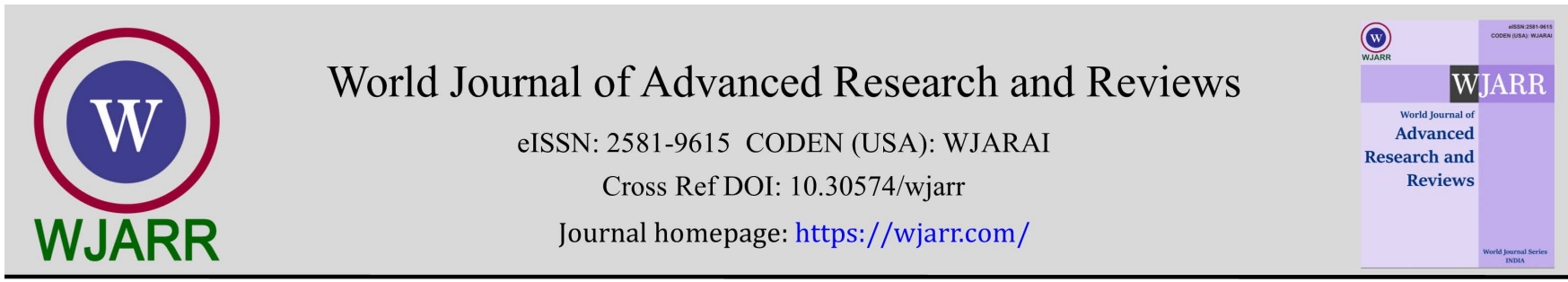

(RESEARCH ARTICLE)

Check for updates

\title{
Non-aspirin NSAIDs use is associated with lowering of liver fibrosis scores in patients with fatty liver disease
}

\author{
Ikechukwu Ronald Ogbu 1, Hossein Akhondi asl 2, Napatkamon Ayutyanont ${ }^{3}$, Andre Manov ${ }^{4}$ and Vignan \\ Manne 5, \\ ${ }^{1}$ Internal Medicine Department, Mountain View Hospital Sunrise Health Consortium, Las Vegas, NV. \\ 2 Associate Program Director, Mountain View Hospital Associate Professor of Medicine, University of Nevada. \\ ${ }^{3}$ Division Director of Research HCA Far West Division, Las Vegas, $N$ V. \\ ${ }^{4}$ Associate Program Director, Mountain View Hospital Professor of Medicine, TCU and UNTHSC School of Medicine, Fort \\ Worth, TX. \\ ${ }^{5}$ Gastroenterology Fellowship Department, Southern Hills Hospital and Medical Center, Las Vegas, NV.
}

World Journal of Advanced Research and Reviews, 2021, 10(01), 230-236

Publication history: Received on 14 March 2021; revised on 19 April 2021; accepted on 21 April 2021

Article DOI: https://doi.org/10.30574/wjarr.2021.10.1.0165

\begin{abstract}
Only a few studies are available with appropriate data on the effects of non-aspirin, non-steroidal anti-inflammatory drugs (NSAIDs) use in patients with fatty liver disease. We performed a retrospective study of 1347 patients with imaging studies that showed fatty liver disease from 2016 through 2019 . We then determined the change in validated indices using Fibrosis-4 (FIB4) and NAFLD fibrosis score (NFS). Patient clinical information, including NSAIDs use, was collected at baseline and then yearly. Using generalized linear models, we estimated the association between nonaspirin NSAIDs use and change in baseline indices. Non-aspirin NSAIDs use was found to be associated with significant lowering of FIB-4 score (0.596 units lower, p-value <0.0001) and NFS (0.431 units lower, p-value 0.0027) every year.

In this retrospective study of patients with fatty liver disease found on imaging, non-aspirin NSAID use was associated with lowering of fibrosis scores, suggesting that NSAID use might be associated with a lower risk for advanced fibrosis in fatty liver disease.
\end{abstract}

Keywords: Fatty liver disease; NSAIDs; Aspirin; Ibuprofen; liver fibrosis; Advance liver disease, Cirrhosis, liver fibrosis indices, NAFLD score, Fibrosis-4 score

\section{Introduction}

Fatty liver disease is the presence of fatty infiltration of the liver. Over time, about $10-25 \%$ of patients with fatty liver disease will progress to advanced fibrosis, cirrhosis, hepatocellular carcinoma, and death [1]. The gold standard to diagnose fatty liver disease and fibrosis is by liver biopsy. However, because the method is invasive, some validated scoring systems are often used to predict the stage of liver fibrosis that could be seen if biopsy was performed. An example of such scoring systems includes Aspartate aminotransferase (AST) to Platelet Ratio (APRI), Fibrosis-4 (FIB4) and NAFLD fibrosis score (NFS) [2].

Hepatic steatosis is usually seen in imaging studies with liver ultrasound, abdominal computed tomography or ProtonMagnetic resonance spectroscopy. When available, magnetic resonance elastography, or vibration controlled elastography can also be used to determine the severity (stage) of fibrosis [3].

\footnotetext{
* Corresponding author: Andre Manov

Associate Program Director, Mountain View Hospital Professor of Medicine, TCU and UNTHSC School of Medicine, Fort Worth, TX. Copyright (C) 2021 Author(s) retain the copyright of this article. This article is published under the terms of the Creative Commons Attribution Liscense 4.0.
} 
In one prospective cohort study of 361 biopsy-proven Nonalcoholic fatty liver disease (NAFLD) patients, daily aspirin use was associated with less risk of progression to fibrosis when compared with non-daily use [4]. In the same study, the use of non-aspirin NSAIDs was not associated with risk for advanced fibrosis, although this was not the primary objective in that study. Another cross-sectional study of 1856 US adult patients with the suspected chronic liver disease showed that aspirin users had lower indices (FIB4, APRI, and NFS) of liver fibrosis among them [5]. Finally, in a murine model study, the result suggested that antiplatelets inhibit the platelet-derived growth factor- $\beta$, which activates the hepatic stellate cells responsible for hepatic fibrosis [6]. It is known that aspirin has an irreversible antiplatelet effect, while other NSAIDs like ibuprofen have a reversible antiplatelet effect.

Despite the growing incidence and burden of fatty liver disease in the USA - 25\% in general population and 56\% in patients with Diabetes Mellitus type 2 and non-alcoholic steatohepatitis (NASH) with Cirrhosis being the second most common cause of liver transplantation in USA, there are no FDA approved medication to prevent the progression of fatty liver disease or reverse the steatosis, fibrosis and inflammation in these patient populations. The data supporting the potential hepatoprotective effect of NSAIDs in liver disease are limited. Therefore, we investigated the association between non-aspirin NSAIDs use and risk of fatty liver progression to advanced fibrosis.

\section{Material and methods}

\subsection{Study Population}

Patients 18 years of age or older in the HCA Healthcare databank system (180 hospitals) with imaging studies that showed fatty liver from January 2016 to January 2019. Imaging studies suggesting fatty liver includes Computed tomography (CT), ultrasonography (US), and magnetic resonance imaging (MRI). Sunrise Hospital-Mountainview Institutional Review Board approved this study with a waiver of documented informed consent.

All patients were de-identified, and the following variables were collected at their baseline and subsequent visits: demographic (age, gender, and race), Medication [aspirin, non-aspirin NSAIDs (ibuprofen, naproxen, ketoprofen, diclofenac, and indomethacin), Statins (atorvastatin, simvastatin, and rosuvastatin)], comorbidities [coronary artery disease (CAD), congestive heart failure (CHF), diabetes mellitus (DM)], and laboratory parameters required for the calculation of the validated non-invasive indices of liver fibrosis FIB4 and NFS [7-8]. We did not use magnetic resonance elastography or vibration controlled elastography to determine the magnitude of the stage of liver fibrosis.

We included only patients who had the needed data and variables to calculate the validated indices every year. We also excluded patients with the following history: unclear or heavy alcohol use history, documented history of alcoholic hepatitis, cirrhosis, hemochromatosis, Wilson's disease, polycystic ovary syndrome, sleep apnea, hypothyroidism, viral hepatitis, autoimmune hepatitis, drug-associated hepatitis, and hepatocellular carcinoma. Based on these criteria, we identified 1347 patients with fatty liver disease.

\subsection{NSAIDs use}

Aspirin and non-aspirin use (ibuprofen, naproxen, ketoprofen, diclofenac, indomethacin) were confirmed by medical record review at every subsequent hospitalization. Only patients who continued to report use at all hospitalization from the year 2016 to 2019 were included. In our cohort, the cited reasons and associated diagnoses for NSAID use included CHF (0.15\%), CAD (0\%), and others (99.85\%).

Radiologic diagnosis of fatty liver disease

US: hyperechoic texture, fatty infiltration, increased echogenicity, hepatomegaly, intra-hepatic vascular blurring (sensitivity $85 \%$, specificity 94\%, decrease sensitivity in morbid obesity) [9-10-11].

CT: reduced attenuation in the liver parenchyma, intrahepatic fat accumulation (Sensitivity $82 \%$, Specificity $100 \%$, noncontrast is more specific)

MRI: hepatic steatosis (Sensitivity 76\%, Specificity 87\%) [11-12].

\subsection{Fibrosis score}

Advanced fibrosis is a known prognostic factor of poor outcomes, but determining fibrosis with biopsy is limited because of the invasive nature. Thus, non-invasive screening indices that only require clinical parameters are often used 
for risk stratification. Using a lower cutoff value of 1.45, a FIB4 score of less or equal to 1.45 correlates with a negative predictive value of $90 \%$, while a FIB4 cutoff value of greater or equal to 3.25 have $97 \%$ specificity. NFS cutoff value of less than or equal to -1.455 has a negative predictive value of $88 \%$ while cutoff value that is greater or equal to 0.676 has a positive predictive value for advanced fibrosis of $82 \%$ [11].

\subsection{Outcomes}

All included patients had sufficient laboratory testing and clinical data needed to calculate NFS or FIB-4 scores each study year. Patients who had missing clinical or laboratory data were excluded from the cohort. Patients were excluded from analysis if their baseline FIB-4 score was greater or equal to 1.45 or if baseline NFS score was equal or greater than $>0.67$. The primary outcomes were defined as an increase or decrease from baseline fibrosis scores. A secondary analysis was performed in different race, gender, and with diabetes type 2 patients.

\subsection{Statistical analysis}

Data are expressed as means or numbers $(\mathrm{N})$ with percentages. To test for normality for age between non-aspirin NSAID use, we used Shapiro-Wilk Test and the Kruskal-Wallis test. A generalized linear model was used to determine the mean indices score at baseline and each subsequent year.

\section{Results}

\subsection{Baseline Characteristics}

Table 1 Describes the Baseline characteristics of patients in the study $(n=1347)$.

Table 1 Baseline clinical and demographic characteristics.

\begin{tabular}{|c|c|}
\hline Characteristics & $N=1347(100 \%)$ \\
\hline Mean Age & 40 \\
\hline \multicolumn{2}{|l|}{ Gender } \\
\hline Male & $504(37 \%)$ \\
\hline Female & $843(63 \%)$ \\
\hline Mean BMI & 34 \\
\hline \multicolumn{2}{|l|}{ Ethnicities } \\
\hline Black & $114(9 \%)$ \\
\hline White & $788(58 \%)$ \\
\hline Other & $445(33 \%)$ \\
\hline \multicolumn{2}{|l|}{ NSAIDs Use } \\
\hline Aspirin & $138(10 \%)$ \\
\hline Non-Aspirin & $1209(90 \%)$ \\
\hline \multicolumn{2}{|l|}{ Medical Conditions } \\
\hline Non DM & $1123(83 \%)$ \\
\hline $\mathrm{DM}$ & $224(17 \%)$ \\
\hline $\mathrm{CHF}$ & $2(<1 \%)$ \\
\hline CAD & $0(0 \%)$ \\
\hline HLD & $0(0 \%)$ \\
\hline \multicolumn{2}{|c|}{ Mean Baseline score of Cohort } \\
\hline NFS & -1.94 \\
\hline FIB-4 & 1.10 \\
\hline
\end{tabular}

Abbreviations: NFS, NAFLD Fibrosis Score; fib-4, Fibrosis-4; BMI, Body Mass Index; NSAIDs, Nonsteroidal Anti-Inflammatory Drugs; DM, diabetes mellitus; CHF, Congestive Heart Failure; CAD, Coronary Artery Disease; HLD, Hyperlipidemia. 


\subsection{Model Analysis of different Parameters}

Table 2 Describes the change in validated scores based on different parameters. NSAIDs use was associated with lowering of validated indices ( $\mathrm{p}$-value $<0.05$ ).

Table 2 Model Analysis of different Parameters.

\begin{tabular}{|l|c|c|}
\hline Parameters & Model Analysis & p-Value \\
\hline NSAIDs Use (N) & & 0.039 \\
\hline Non-DM & 1123 & \\
\hline DM & 224 & \\
\hline FIB-4 & Change in Mean Baseline Score & \\
\hline Gender & -0.051 & 0.57 \\
\hline NSAIDs use & -0.596 & $<0.0001$ \\
\hline Black & -0.433 & 0.42 \\
\hline Other & -0.054 & 0.62 \\
\hline Hispanics & -0.277 & 0.0099 \\
\hline NFS & Change in Mean Baseline Score & \\
\hline Gender & -0.068 & 0.44 \\
\hline NSAIDs use & -0.431 & 0.0027 \\
\hline Black & 0.194 & 0.23 \\
\hline Other & -0.234 & 0.03 \\
\hline Hispanics & -0.231 & 0.03 \\
\hline
\end{tabular}

Abbreviations: NFS, NAFLD Fibrosis Score; fib-4, Fibrosis-4; NSAIDs, Nonsteroidal Anti-Inflammatory Drugs; DM, diabetes mellitus.

Table 3 compares histological staging with corresponding noninvasive scores.

Table 3 Fibrosis Severity Scale and corresponding noninvasive staging Cohort Mean indices at baseline (FIB-4: 1.10; NFS: -1.94).

\begin{tabular}{|l|l|l|}
\hline Histologic Stage & FIB4-Index & NAFLD Score \\
\hline F0-F1 No Fibrosis or Mild Fibrosis & $<1.45$ & $<-1.455$ \\
\hline F1-F2 Moderate Fibrosis & $1.45-3.25$ & $-1.455-0.675$ indeterminate score \\
\hline F3-F4- Severe Fibrosis or Cirrhosis & $>3.25$ & $>0.675$ \\
\hline
\end{tabular}

Noninvasive tests of hepatic fibrosis are used to predict the stage of liver fibrosis that could be seen if biopsy was performed.

The cohort in this study had a Baseline mean FIB4 of 1.10 and mean NFS of -1.94 which corresponds with no fibrosis or mild fibrosis at baseline.

Figure 1 Generalized Linear model analysis.

Non-aspirin NSAIDs use was associated with reduction in mean indices score during each study year $(p$-value $<0.05)$. 


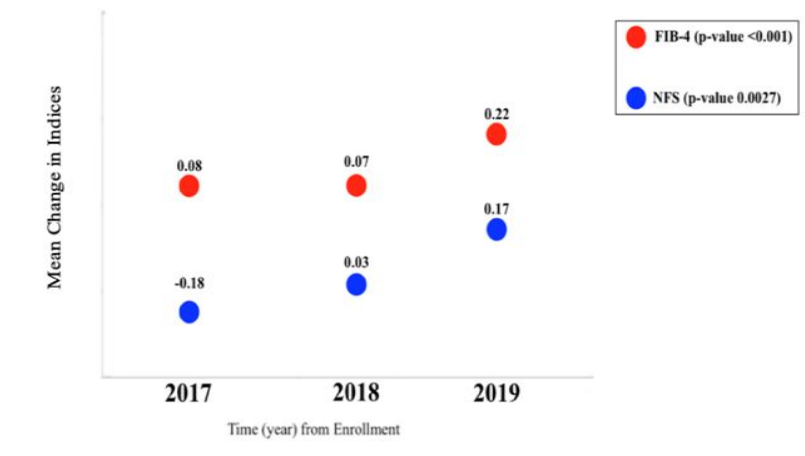

Abbreviations: FIB-4, Fibrosis-4; NFS, NAFLD Fibrosis Score.

Figure 1 Change in validated scores with non-aspirin NSAID use.

\section{Discussion}

In our retrospective study of patients with fatty liver disease seen on imaging studies, non-aspirin NSAIDs use was associated with lowering of FIB-4 and NFS scores every year. Majority of the patients in the study were taking nonaspirin NSAIDs for non-cardiac reasons, which suggest that any observed changes was mostly due to non-aspirin effect. In another prospective study, similar findings were reported in biopsy-proven NAFLD patients who were taking aspirin daily [5]. The clinical data needed to fully support the hepato-protective effect of aspirin or other non-aspirin NSAIDs is still limited. The current study adds a piece of retrospective evidence to support the potential benefit of NSAIDs in fatty liver disease.

The observed benefit could be due to mechanisms previously described in murine models, which suggest that platelets stimulate the stellate cells to initiate fibrosis via platelet-derived growth factor (PDGF). NSAIDs with reversible or irreversible antiplatelet effect could potentially prevent such hepatic fibrosis by inhibition of PDGF [6].

Given the prevalence of fatty liver disease in the United States and worldwide (10-46\%), NSAIDs potential benefit could be profound in these patient population. Thus, further research is needed to better understand the potential benefit and bleeding risk of NSAIDs.

We would like to mention that there have being studies in the literature with Pioglitazone in patients with NAFL/NASH confirmed on liver biopsy in patients with or without Diabetes mellitus type 2 which showed complete resolution of NASH in $51 \%$ of the patients with placebo subtracted difference of $32 \%$ [3]. This improvement correlated with improvement of adipose tissue insulin resistance and increased adiponectin level. Similar resolution of NAFL/NASH was observed in patients with Diabetes Mellitus type 2 treated with Liraglutide. 39\% of patients had resolution of NASH. This effects were related to improving the body weight and, glycemic control and insulin sensitivity with Liraglutide [3].

We acknowledge that there might be limitations to this study, which are not limited to the following:

- Fatty liver diagnosis was based on imaging reports of different modalities, which could add variability in the observed results.

- Because we did not use magnetic resonance elastography or vibration controlled elastography to determine the severity of liver fibrosis, some patients in the cohort could potentially have different degree of fibrosis at baseline

- Differences in medication dose and duration of use was not accounted for in this study.

- The length of the study is not sufficient to fully determine the clinical significance of lowered fibrosis scores.

- Recall bias and exposure misclassification was not accounted for in this study. However, we tried to minimize this risk by careful examination of medical records.

- The presence of other conditions not accounted for or not yet diagnosed may also cause confounding in the observed study results.

- Non-invasive indices are not the gold standard for evaluating hepatic fibrosis; however, they have demonstrated utility for this purpose. 


\section{Conclusion}

Non-aspirin NSAIDs use is associated with lowering of hepatic fibrosis scores. In a group of patients with fatty liver disease on imaging, non-aspirin NSAIDs use was associated with significant change in validated fibrosis indices.

\section{Compliance with ethical standards}

\section{Acknowledgments}

This research was supported (in whole or in part) by HCA Healthcare and/or an HCA Healthcare affiliated entity. The views expressed in this publication represent those of the authors and do not necessarily represent the official views of HCA Healthcare or any of its affiliated entities.

\section{Disclosure of conflict of interest}

None to be disclosed for any of the authors.

\section{Statement of informed consent}

Informed consent was obtained from all individual participants included in the study.

\section{References}

[1] Wong RJ, Liu B, Phuket T. Significant burden of non-alcoholic fatty liver disease with advanced fibrosis in the US: a cross-sectional analysis of 2011-2014 National Health and Nutrition Examination Survey. Aliment Pharmacol Ther. 2017 Nov; 46(10): 974-980.

[2] Hagstrom H, Nasr P, Ekstedt, et al. Accuracy of non-invasive scoring systems in assessing risk of death and liverrelated endpoints in patients with non-alcoholic fatty liver disease. Clinical gastroenterology and hepatology. 2019; 17: 1148-1156.

[3] Barb, Diana. Pharmacological approaches to the patient with Nonalcoholic Fatty Liver Disease. 2020 Endocrine Case management: Meet the professor. 2020; 121-135.

[4] Simon TG, Henson J, Osganian S, Masia R, Chan AT, Chung RT, Corey KE. Daily Aspirin Use Associated with Reduced Risk for Fibrosis Progression in Patients with Nonalcoholic Fatty Liver Disease. Clin Gastroenterol Hepatol. 2019 Dec; 17(13): 2776-2784.e4.

[5] Jiang ZG, Feldbrügge L, Tapper EB, Popov Y, Ghaziani T, Afdhal N, Robson SC, Mukamal KJ. Aspirin use is associated with lower indices of liver fibrosis among adults in the United States. Aliment Pharmacol Ther. 2016 Mar; 43(6): 734-43.

[6] Yoshida S, Ikenaga N, Liu SB, Peng ZW, Chung J, Sverdlov DY, Miyamoto M, Kim YO, Ogawa S, Arch RH, Schuppan D, Popov Y. Extrahepatic platelet-derived growth factor- $\beta$, delivered by platelets, promotes activation of hepatic stellate cells and biliary fibrosis in mice. Gastroenterology. 2014 Dec; 147(6): 1378-92.

[7] Sterling RK, Lissen E, Clumeck N, Sola R, Correa MC, Montaner J, S Sulkowski M, Torriani FJ, Dieterich DT, Thomas DL, Messinger D, Nelson M; APRICOT Clinical Investigators. Development of a simple non-invasive index to predict significant fibrosis in patients with HIV/HCV coinfection. Hepatology. 2006 Jun; 43(6): 1317-25.

[8] Xiao G, Zhu S, Xiao X, Yan L, Yang J, Wu G. Comparison of laboratory tests, ultrasound, or magnetic resonance elastography to detect fibrosis in patients with non-alcoholic fatty liver disease: A meta-analysis. Hepatology. 2017 Nov; 66 (5): 1486-1501.

[9] Hernaez R, Lazo M, Bonekamp S, Kamel I, Brancati FL, Guallar E, Clark JM. Diagnostic accuracy and reliability of ultrasonography for the detection of fatty liver: a meta-analysis. Hepatology. 2011 Sep 2; 54(3): 1082-1090.

[10] Cho CS, Curran S, Schwartz LH, Kooby DA, Klimstra DS, Shia J, Munoz A, Fong Y, Jarnagin WR, DeMatteo RP, Blumgart LH, D'Angelica MI. Preoperative radiographic assessment of hepatic steatosis with histologic correlation. J Am Coll Surg. 2008 Mar; 206(3): 480-8.

[11] Li Q, Dhyani M, Grajo JR, Sirlin C, Samir AE. Status of imaging in non-alcoholic fatty liver disease. World J Hepatol. 2018 Aug 27; 10(8): 530-542. 
World Journal of Advanced Research and Reviews, 2021, 10(01), 230-236

[12] Vernon G, Baranova A, Younossi ZM. Systematic review: the epidemiology and natural history of non-alcoholic fatty liver disease and non-alcoholic steatohepatitis in adults. Aliment Pharmacol Ther. 2011 Aug; 34 (3): 27485. 